A Contribuição dos Produtos Naturais como Fonte de Novos Fármacos Anticâncer: Estudos no Laboratório Nacional de Oncologia Experimental da Universidade Federal do Ceará

\author{
Costa-Lotufo, L. V.; Montenegro, R. C.; Alves, A. P. N. N., Madeira, S. V. F.; Pessoa, C.; \\ Moraes, M. E. A.; Moraes, M. O.
}

Rev. Virtual Quim., 2010, 2 (1), 47-58. Data de publicação na Web: 30 de agosto de 2010

http://www.uff.br/rvq

\title{
The Contribution of Natural Products as Source of New Anticancer Drugs: Studies Carried Out at the National Experimental Oncology Laboratory from the Federal University of Ceará
}

\begin{abstract}
The history of anticancer chemotherapy is closely related to that of natural products, as over $60 \%$ of currently used drugs in cancer therapy are natural products or inspired by a natural compound. Despite the introduction of new drugs in the therapeutic arsenal of anticancer medicines, many tumors do not properly respond to treatment. Natural resources comprise the most promising possibilities of finding new and efficient molecules with impact in the therapy of resistant diseases. The National Experimental Oncology Laboratory began a research program aiming at the evaluation of new natural or synthetic anticancer molecules through an experimental approach involving in vitro and in vivo models. In the period between 2000 and 2007, 5166 samples were screened, and three molecules, piplartine, biflorine and pisosterol, were selected for further studies.
\end{abstract}

Keywords: natural products; anticancer activity; biodiversity

\section{Resumo}

A história dos medicamentos anticâncer está intimamente relacionada aos produtos naturais, sendo que mais de $60 \%$ dos medicamentos utilizados tem em alguma instância sua origem relacionada a uma fonte natural. Apesar da introdução de novos fármacos no arsenal terapêutico do câncer, vários tumores ainda não dispõem de tratamento adequado. As fontes naturais ainda estão disponíveis em abundância e oferecem as melhores possibilidades de encontrar substâncias de interesse terapêutico. O Laboratório Nacional de Oncologia Experimental estabeleceu um programa com o objetivo de avaliar o potencial anticâncer de compostos obtidos da Caatinga, Mata Atlântica, Amazônia, Cerrado e Ecossistemas Marinhos, além de compostos obtidos através de síntese. Esses estudos baseiam-se na determinação da atividade antitumoral in vitro e in vivo. Das 5166 amostras avaliadas no período de 2000 a 2007, foram selecionadas a piplartina, a biflorina e o pisosterol para a continuidade dos estudos como antitumorais.

palavras-chave: produtos naturais; atividade anticâncer; biodiversidade

\footnotetext{
* Departamento de Fisiologia e Farmacologia, Rua Cel Nunes de Melo 1127, Rodolfo Teófilo Fortaleza, 60430270, CE, Brasil.

M Ivcosta@ufc.br
} 


\title{
A Contribuição dos Produtos Naturais como Fonte de Novos Fármacos Anticâncer: Estudos no Laboratório Nacional de Oncologia Experimental da Universidade Federal do Ceará
}

\author{
Letícia V. Costa-Lotufo,* Raquel C. Montenegro, Ana Paula N. N. Alves, Socorro V. F. \\ Madeira, Cláudia Pessoa, Maria E. A. de Moraes, Manoel O. de Moraes
}

LOE, Laboratório Nacional de Oncologia Experimental, Departamento de Fisiologia e Farmacologia, Faculdade de Medicina, Universidade Federal do Ceará, Fortaleza, Ceará, Rua Cel. Nunes de Melo 1127, 60430-270,

Fortaleza, CE, Brasil.

*Ivcosta@ufc.br

Recebido em 25 de junho de 2009. Aceito para publicação em 16 de outubro de 2009

\section{Introdução}

\section{Estado da Arte}

\section{O Programa de P\&D de Novos Fármacos Anticâncer do LOE-UFC}

\section{Moléculas Promissoras}

\section{Conclusões}

\section{Introdução}

Os tumores malignos são responsáveis por um número expressivo e crescente de pacientes em todo o mundo, e representam a segunda causa de morte da população mundial. ${ }^{1}$ No Brasil, estima-se em 500 mil o número de novos casos em 2009, e uma mortalidade que deverá atingir mais de 250 mil portadores de câncer. ${ }^{2}$

A terapêutica do câncer baseia-se, de forma geral, na associação da ressecção cirúrgica dos tumores ao tratamento radioterápico, e a quimioterapia. $\mathrm{Na}$ década de setenta estas formas clássicas de abordagem sofreram alterações significativas com a introdução do conceito de tratamento adjuvante. 0 emprego intensivo dos protocolos pós-cirúrgicos, incluindo a associação de quimioterápicos com diferentes mecanismos de ação à radioterapia e, mais recentemente, a associação destes aos anticorpos monoclonais, vem melhorando os resultados do tratamento de alguns tipos de cânceres. Infelizmente, muitos tumores ainda apresentam respostas modestas aos protocolos clínicos, limitando a indicação e a eficácia do tratamento adjuvante tanto para os tumores primários quanto para as metástases. $^{3}$ Portanto, torna-se imperativo a necessidade de encontrar, desenvolver, e introduzir no arsenal médico, modalidades terapêuticas mais eficientes que possam oferecer ao número crescente de pacientes com doenças malignas disseminadas, oportunidades reais de controle locorregional e a distância das células neoplásicas.

$O$ século $X X$ apresentou um avanço extraordinário na pesquisa de produtos naturais, especialmente de plantas e microorganismos, no campo da oncologia propiciando a descoberta de diversas substâncias utilizadas atualmente na terapêutica antineoplásica. A maioria (60\%) dos fármacos anticâncer introduzida na terapêutica nas últimas décadas tem sua origem nos produtos naturais. ${ }^{4-6}$ Dentre estes se destacam a vimblastina (1, Velban ${ }^{\circledR}$ ) e a vincristina (2, Oncovin $\left.{ }^{\circledR}\right)$ e os análogos vindesina (3, Eldisine ${ }^{\circledR}$ ) e vinorelbina (4, Navelbine $\left.{ }^{\circledR}\right)$; o paclitaxel $\left(5\right.$, Taxol$\left.^{\circledR}\right)$ e o análogo docetaxel (6, Taxotere ${ }^{\circledR}$ ); a podofilotoxina (7) e os análogos, etoposídeo (8, Etopophos ${ }^{\circledR}$ ) e teniposídeo $\left(9\right.$, Vumon $\left.^{\circledR}\right)$; e a camptotecina (10) e os análogos, 
topotecano (11, Hycamtin $\left.{ }^{\circledast}\right)$ e irinotecano (12, Camptosar ${ }^{\circledast}$ ) (Figura 1). Estes medicamentos movimentam anualmente um mercado de cerca de 60 bilhões de dólares. $^{7}$

As fontes naturais ainda estão disponíveis em abundância e oferecem as melhores possibilidades de encontrar substâncias de interesse terapêutico. De fato, mais de uma centena de compostos derivados de produtos naturais está em fase de testes clínicos, principalmente para tratamento do câncer e de doenças infecciosas. ${ }^{6}$ Além disso, um total de 13 fármacos derivados de produtos naturais foram aprovados para utilização clínica entre 2005 e $2007 .{ }^{5}$

Apesar da introdução de novos fármacos no arsenal terapêutico contra o câncer, vários tumores sólidos ainda não dispõem de tratamento adequado. O carcinoma de pulmão de não-pequenas células que se encontra entre os mais frequentes em todo o mundo, apresenta respostas modestas a todos os esquemas quimioterápicos disponíveis. Como a monoterapia apresenta apenas resposta parcial em $15 \%$ a $20 \%$ dos casos e com as associações terapêuticas não ultrapassa de $40 \%$ a $50 \%$, é necessária a busca de novas alternativas medicamentosas para melhorar a eficácia do tratamento de doenças neoplásicas avançadas. Por outro lado, a morbidade associada aos quimioterápicos ainda é um obstáculo significativo. A descoberta de fármacos antineoplásicos de fácil administração e com poucos ou insignificantes efeitos colaterais é uma das principais metas buscadas pelos pesquisadores da área.

A maioria dos quimioterápicos usados na terapêutica foi selecionada por sua capacidade de controlar a proliferação celular. ${ }^{8}$ Entretanto, recentemente, foram identificados fármacos com atividade específica contra alguns mecanismos metabólicos da célula tumoral. Também vêm sendo pesquisados, com sucesso, fármacos que inibem a neovascularização tumoral, que induzem a rediferenciação celular ou que levem a célula tumoral a apoptose. Desta forma, as moléculas pesquisadas precisam ser testadas também nesses novos alvos terapêuticos.

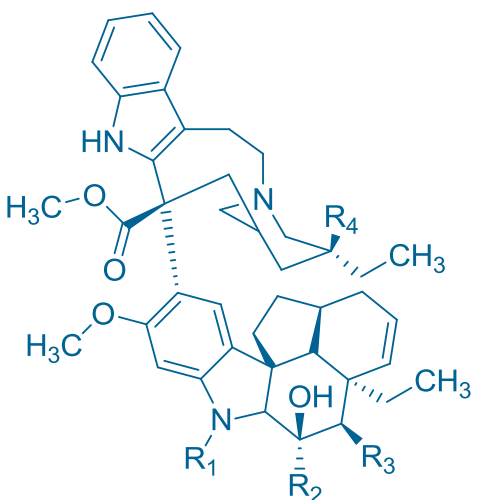

$\mathrm{R}_{1}=\mathrm{CH}_{3} ; \mathrm{R}_{2}=\mathrm{CO}_{2} \mathrm{CH}_{3} ; \mathrm{R}_{3}=\mathrm{OCH}_{2} \mathrm{CH}_{3} ; \mathrm{R}_{4}=\mathrm{OH}$ (1)

$\mathrm{R}_{1}=\mathrm{COH} ; \mathrm{R}_{2}=\mathrm{CO}_{2} \mathrm{CH}_{3} ; \mathrm{R}_{3}=\mathrm{OCH}_{2} \mathrm{CH}_{3} ; \mathrm{R}_{4}=\mathrm{OH}$ (2)

$\mathrm{R}_{1}=\mathrm{CH}_{3} ; \mathrm{R}_{2}=\mathrm{CONH}_{2} ; \mathrm{R}_{3}=\mathrm{OH} ; \mathrm{R}_{4}=\mathrm{OH}(3)$

$\mathrm{R}_{1}=\mathrm{CH}_{3} ; \mathrm{R}_{2}=\mathrm{CO}_{2} \mathrm{CH}_{3} ; \mathrm{R}_{3}=\mathrm{OCH}_{2} \mathrm{CH}_{3} ; \mathrm{R}_{4}=\mathrm{H}$ (4)

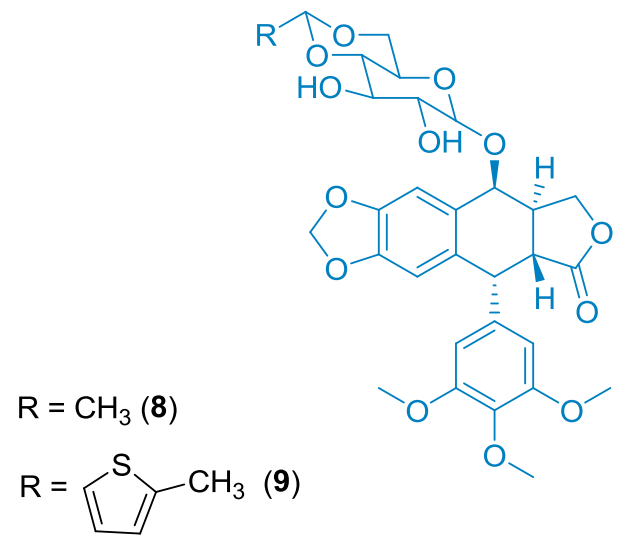

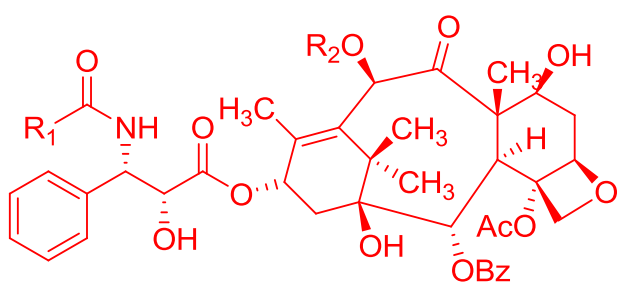

$\mathrm{R}_{1}=\mathrm{Ph} ; \mathrm{R}_{2}=\mathrm{COCH}_{3}(\mathbf{5})$

$\mathrm{R}_{1}=\mathrm{OC}\left(\mathrm{CH}_{3}\right)_{3} ; \mathrm{R}_{2}=\mathrm{H}(6)$<smiles>COc1cc([C@H]2c3cc4c(cc3[C@@H](O)[C@@H]3COC(=O)[C@H]23)OCO4)cc(OC)c1OC</smiles>

(7)

Figura 1. Fármacos derivados de plantas usados na terapia do câncer 


\section{Estado da Arte}

O Câncer compreende um conjunto de doenças que se caracterizam pela presença de células em crescimento contínuo, com propriedades de invasão e destruição do tecido adjacente, bem como, de crescimento em outros sítios diferentes do tumor primário (metastização). ${ }^{9} \quad$ Essas propriedades geralmente são ocasionadas por um acúmulo de mutações nos oncogenes, nos genes supressores e nos genes reparadores de DNA, o que caracteriza o câncer como doença genética. ${ }^{10}$ De um modo geral, os oncogenes promovem a proliferação celular ordenada enquanto os genes supressores mantêm essa proliferação sob controle restringindo o crescimento celular. Os genes reparadores de danos do DNA estão constantemente refazendo as moléculas que sofrem mutação. $O$ mau funcionamento dos mecanismos de regulação do ciclo celular permite a passagem das células mutadas pelo ciclo, acumulando mutações que contribuem para o surgimento das características do tumor maligno. ${ }^{11,12}$ O câncer afeta, portanto, a população de um modo geral, incluindo pessoas de ambos os sexos e de todas as idades.

Nos últimos anos, a quimioterapia tem conseguido êxitos notáveis na cura de algumas formas de cânceres disseminados tais como a leucemia aguda infantil, distintos tipos de linfomas e alguns tipos de tumores sólidos, em especial os derivados de células germinais. Ao contrário, a melhora no tratamento sistêmico de tumores sólidos mais freqüentes em adultos (pulmão, mama, cólon e pâncreas) não sofreu grandes avanços, resultando em altos índices de mortalidade dentre os pacientes. Há, portanto, uma clara e urgente necessidade de identificar, avaliar e desenvolver novos e mais eficientes fármacos para o tratamento desses cânceres. ${ }^{13}$

$O$ atual interesse na busca de novos agentes antimitóticos, por exemplo, é conseqüência de sua importância para o tratamento de diferentes formas de tumores malignos. Alguns fármacos em uso corrente na terapia do câncer foram descobertos de forma racional, baseada no desenho da estrutura, porém, a grande maioria foi descoberta por processos empíricos. O início, na década de quarenta, de programas de prospecção de fármacos com atividade antineoplásica resultou na descoberta dos compostos de mostarda nitrogenada. Hoje existem mais de uma centena de fármacos ativos no tratamento do câncer, muitos dos quais obtidos em programas de bioprospecção. Uma proporção importante dos fármacos antitumorais atualmente utilizados em clínica foi obtida a partir de produtos naturais. A utilização por Farber em 1954 de um antibiótico extraído do cultivo de uma espécie de Streptomyces, a Actinomicina $\mathrm{D}$, para tratar um paciente com tumor de Wilms metastático, introduziu no tratamento de câncer o primeiro fármaco antineoplásico derivado de um produto natural, despertando grande interesse no meio científico nessa área de pesquisa, o qual perdura até hoje.

É importante ressaltar que muitas das suposições quanto à eficácia dos produtos naturais utilizados pela medicina tradicional ainda são vistas com certo ceticismo. ${ }^{14}$ Ainda assim, alguns compostos naturais foram identificados em função do conhecimento etnofarmacológico de algumas plantas. A tabela 1 resume as principais classes desses fitoquímicos e seus derivados que atualmente são utilizados na quimioterapia do câncer, seus alvos moleculares e indicações terapêuticas. ${ }^{8,15,16}$

As estratégias para a descoberta de novos fármacos têm mudado ao longo dos anos. Os programas de prospecção no Instituto Nacional do Câncer dos Estados Unidos começaram no início dos anos 50 e consistiam em testes de novos compostos em camundongos inoculados com leucemias L1210 e P388. Esse modelo foi bastante questionado, uma vez que não era considerado representativo dos tumores humanos, na sua maioria sólidos. ${ }^{17}$ Sendo assim, o modelo foi reconsiderado e, atualmente, os programas de prospecção incluem uma etapa inicial de testes in vitro em linhagens tumorais humanas utilizando técnicas automatizadas (High Troughtput Screening - HTS) o que tem resultado num elevado número de moléculas promissoras. ${ }^{18,19}$

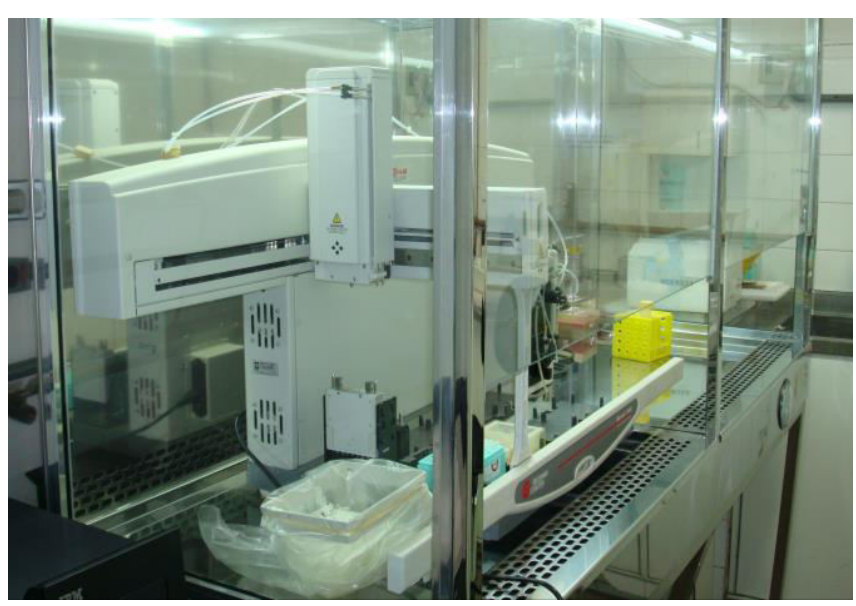

Figura 2. Estação automatizada (High Throughtput Screening - HTS) utilizada nos ensaios de citotoxicidade no Laboratório Nacional de Oncologia Experimental. 
Tabela 1: Produtos naturais ou fármacos derivados de plantas utilizados na terapia do câncer ${ }^{8,15,16}$

\begin{tabular}{|c|c|c|c|}
\hline Fármaco & Fonte & Alvo Molecular & Indicação terapêutica \\
\hline $\begin{array}{l}\text { Vimblastina (1), } \\
\text { vincristina (2), } \\
\text { vindesina (3) e } \\
\text { vinorelbina (4) }\end{array}$ & Catharanthus roseus & Tubulina/ Microtúbulos & $\begin{array}{c}\text { Leucemia linfoblástica } \\
\text { aguda; câncer de } \\
\text { testículo; doença de } \\
\text { Hodking }\end{array}$ \\
\hline $\begin{array}{c}\text { Paclitaxel (5) e } \\
\text { docetaxel (6) }\end{array}$ & Taxus brevifolia & Tubulina/ Microtúbulos & Câncer de mama \\
\hline $\begin{array}{l}\text { Podofilotoxina (7) } \\
\text { Etoposídeo (8) e } \\
\text { teniposídeo (9) }\end{array}$ & Podophyllum peltatum & Topoisomerase II & $\begin{array}{c}\text { Câncer de Pulmão, } \\
\text { ovário e testículo; } \\
\text { Leucemia Linfocítica } \\
\text { Aguda; }\end{array}$ \\
\hline $\begin{array}{l}\text { Camptotecina (10) } \\
\text { Topotecano (11) e } \\
\text { irinotecano (12) }\end{array}$ & $\begin{array}{c}\text { Camptotheca } \\
\text { accuminata }\end{array}$ & Topoisomerase I & Câncer de colon \\
\hline
\end{tabular}

Neste contexto onde milhares de moléculas podem ser testadas num curto período de tempo, a química combinatória ganhou fôlego na indústria e na academia, porém a realidade mostrou sucesso limitado no uso de bibliotecas de compostos sintéticos obtidos através da química combinatória para o desenvolvimento de novos medicamentos. 0 sorafenib, utilizado na terapia da leucemia mielóide aguda, foi a única molécula obtida a partir desta estratégia a atingir o mercado farmacêutico., ${ }^{4,6} \mathrm{O}$ acesso às fontes naturais, por outro lado, comprova que a criatividade da natureza é praticamente insuperável e a evolução tecnológica do HTS é um poderoso aliado nesse processo de descoberta não só de novas moléculas, mas de alvos e estratégias terapêuticas nunca antes pensadas.

De um lado, novas fontes passam a ser exploradas, como actinomicetos marinhos, cianobactérias e fungos endofíticos, muitas vezes graças a evolução de técnicas como, por exemplo, a metagenômica, o que per si multiplica a diversidade de metabólitos isolados. ${ }^{6}$ De outro lado, ou melhor, adicionalmente, a química experimenta uma otimização das técnicas analíticas o que possibilita, segundo alguns autores, o isolamento de compostos bioativos e a elucidação estrutural em períodos muitas vezes inferiores a um mês. ${ }^{20}$

Com a aprovação, em 2007, dos medicamentos Ixempra ${ }^{\circledR}$ (ixabepilona, 13), derivado da epotilona (14) com ação em microtúbulos, Yondelis ${ }^{\circledR}$ (trabectedina,
15), primeiro medicamento anticâncer verdadeiramente de origem marinha, alquilante de DNA, e Torise $^{\circledR}$ (temsirolimus, 16), inibidor de proteína quinase mTOR, os produtos naturais estão definitivamente de volta ao cenário da pesquisa em oncologia. ${ }^{16}$

Existe um potencial extraordinário para a descoberta de novos fármacos anticâncer de ocorrência natural em função da existência de um grande número de espécies disponíveis para investigação. Até o momento, calcula-se que menos de $2 \%$ das plantas superiores foram analisadas para deteç̧ão de constituintes com atividade antineoplásica e mesmo assim, buscando apenas a atividade citotóxica. $\mathrm{Na}$ realidade, uma grande quantidade de moléculas com atividade antineoplásica derivadas de organismos marinhos, microorganismos e de plantas ainda pode ser revelada. ${ }^{21}$ Além disso, o conhecimento de novos alvos terapêuticos no câncer amplia a possibilidade da descoberta de novas moléculas com potencial anticâncer. Neste cenário, o sucesso do desenvolvimento de novas drogas depende fundamentalmente de um esforço multidisciplinar na descoberta de novas moléculas naturais lideres e da otimização molecular através da aplicação de técnicas de síntese, química combinatória e bioquímica, aliadas a estudos biológicos de alta qualidade. ${ }^{22}$ 
<smiles>C/C(=C\c1csc(C)n1)C(C[C@@H]1O[C@]1(C)CCC[C@@H](C)[C@@H](O)[C@@H](C)C(=O)C(C)(C)[C@@H](O)C[C@@H]1O[C@H]1C)NC(=O)C[C@@H]1O[C@H]1C</smiles>

(13)<smiles>CO[C@@H](C[C@@H]1O[C@]1(C)CCC[C@H](C)[C@H](O)[C@H](C)C(=O)C(C)(C)[C@@H](O)CC(=O)O)/C(C)=C/c1csc(C)n1</smiles>

(14)

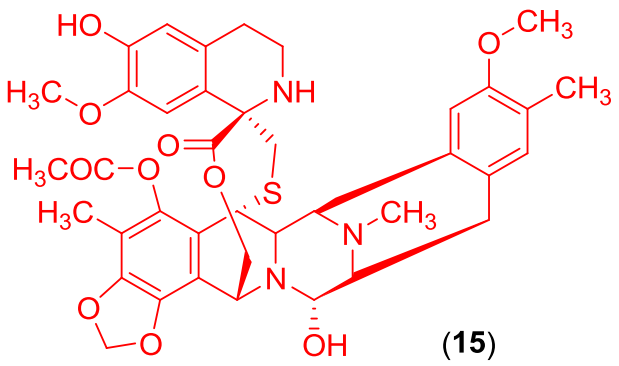

(15)

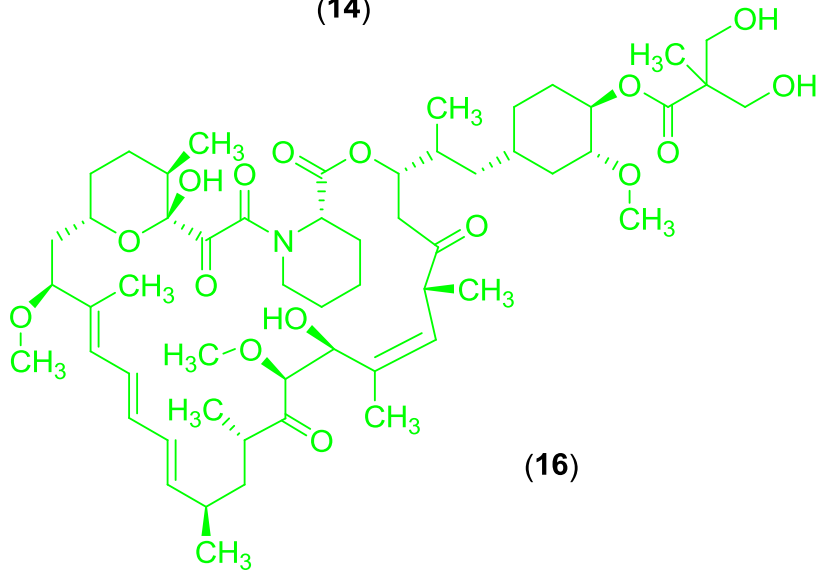

Figura 3. Fármacos derivados de produtos naturais introduzidos em 2007 no mercado farmacêutico.

O Brasil tem uma área de 8,5 milhões $\mathrm{km}^{2}$ e possui várias zonas climáticas que incluem o trópico úmido no Norte, o semiárido no Nordeste e áreas temperadas no Sul. As diferenças climáticas contribuem para as diferenças ecológicas formando zonas biogeográficas distintas chamadas biomas. Dos diversos biomas brasileiros (Floresta Amazônica, Cerrado, Mata Atlântica, Pantanal, Caatinga, Manguezal, etc.), estima-se que existam cerca de 60 mil espécies vegetais. ${ }^{23}$ Segundo Kato, ${ }^{24}$ o Brasil é o país com maior biodiversidade do mundo, contando com $20 \%$ do número total de espécies do planeta, sendo que muitas dessas espécies são endêmicas. A composição total da biodiversidade brasileira não é conhecida e talvez nunca venha a ser, tal a sua magnitude e complexidade.

Assim, o gerenciamento e a promoção do desenvolvimento sustentável dessas regiões constituem-se em tarefa extremamente difícil, complexa e de enorme responsabilidade. A biodiversidade brasileira representa para nossa população e, particularmente para os pesquisadores brasileiros, uma região de enorme importância científica, social, econômica, cultural e ambiental. Em verdade, as pesquisas científicas e tecnológicas são instrumentos essenciais para o desenvolvimento sustentável de um ecossistema tão diversificado e rico. A ocupação da floresta brasileira, a utilização racional dos recursos naturais e o uso do solo só podem acontecer, de forma satisfatória, se estratégias de desenvolvimento científico e tecnológico forem promovidas e especialmente desenhadas para a compreensão dos fenômenos naturais e o papel do homem nesses sistemas.

A mega biodiversidade do Brasil por si justifica o enorme potencial para a descoberta de medicamentos baseados em produtos naturais. Além disso, vários estudos realizados vêm a comprovar esse potencial no que diz respeito à atividade antitumoral. Algumas revisões recentes foram escritas a cerca do potencial anticâncer de produtos oriundos da nossa biodiversidade. $^{25-31}$ Assim sendo, neste trabalho apresentaremos o programa de bioprospecção de novas substâncias ou moléculas ou fármacos anticâncer do Laboratório Nacional de Oncologia Experimental (LOE) da Universidade Federal do Ceará, ressaltando os resultados promissores obtidos.

\section{O Programa de prospecção de novos fármacos anticâncer do LOE-UFC}

A Oncologia Experimental procura estudar os mecanismos de desenvolvimento das neoplasias em modelos experimentais, assim como encontrar as possíveis formas de tratá-las. O LOE vem, desde 1989, realizando pesquisas de prospecção de novas moléculas com potencial atividade antitumoral. Nesse 
sentido, estabeleceu uma extensa colaboração com vários grupos de pesquisa em todo o País. É importante salientar que as universidades e centros de pesquisa são os únicos provedores de moléculas e bases medicamentosas para a indústria farmacêutica nacional.

Todo esse cenário levou a implantação de um programa para bioprospecção e a realização de testes para determinar o potencial anticâncer de moléculas extraídas de plantas, organismos marinhos e microorganismos envolvendo diversas instituições de ensino e pesquisa com vistas à criação da Rede Nacional para Prospecção de Fármacos Anticâncer. Essas colaborações vêm ocorrendo de maneira interativa com o objetivo maior de realizar um estudo multidisciplinar e interinstitucional que visa um conhecimento mais amplo do potencial terapêutico da nossa biodiversidade de maneira sustentável. Desta forma, além das informações sobre a química, farmacologia, etnofarmacologia e quimiossistemática, as colaborações visam também o conhecimento da toxinologia e química de animais marinhos e microorganismos, além do fator multiplicador relacionado à formação de recursos humanos capacitados no âmbito da iniciação científica, mestrado e doutorado. Ressalte-se que o LOE está vinculado ao INCT (Instituto Nacional de Ciência e Tecnologia) de Fármacos e Medicamentos (INCTINOFAR), coordenado pelo professor Eliezer J. Barreiro. É importante também mencionar que está em processo de conclusão o Centro de Pesquisa e Desenvolvimento de Medicamentos da UFC (CPDM) com vistas à realização de pesquisa em toda a cadeia produtiva do medicamento. Sendo assim, a convergência de interesses do LOE, INCT e CPDM aperfeiçoarão esse processo no que diz respeito à pesquisa e ao desenvolvimento de fármacos e medicamentos antineoplásicos.

O programa baseia-se na avaliação da citotoxicidade das amostras em células tumorais humanas. Para esse fim, o LOE conta com um banco de mais de 50 linhagens de células tumorais doadas pelo Instituto Nacional do Câncer dos Estados Unidos (NCl-USA) através de um memorando de transferência tecnológica, assinado em 2001 e renovado em 2006. Na etapa inicial, as amostras das mais diversas origens, naturais ou sintéticas, são submetidas a um teste em concentração única (50 $\mu \mathrm{g} / \mathrm{mL}$ para extratos, $20 \mu \mathrm{g} / \mathrm{mL}$ para frações e 5 $\mu \mathrm{g} / \mathrm{mL}$ para substâncias puras) utilizando três linhagens de tumores sólidos, SF-295 (Sistema Nervoso Central), MDA-MB-435 (Melanoma) e HCT-8 (Cólon), através do método do MTT descrito por Mosmann, em $1983 .^{32}$ Neste momento, são selecionadas as amostras que inibem seletivamente ou indiscriminadamente a proliferação celular.

A partir deste momento, as amostras de extratos, por envolverem misturas complexas são submetidas ao processo de fracionamento e purificação, que quando possível é guiado pela atividade biológica. De posse, então, do composto purificado, procede-se com a caracterização da atividade citotóxica, determinando a eficácia do composto num painel amplo de linhagens, sua seletividade e seu mecanismo de ação. Os ensaios em animais são realizados para comprovação da atividade in vivo quando da disponibilidade de material suficiente, e das evidências experimentais que justifiquem seu uso. o fluxograma de desenvolvimento adotado no programa está esquematizado na Figura 4.

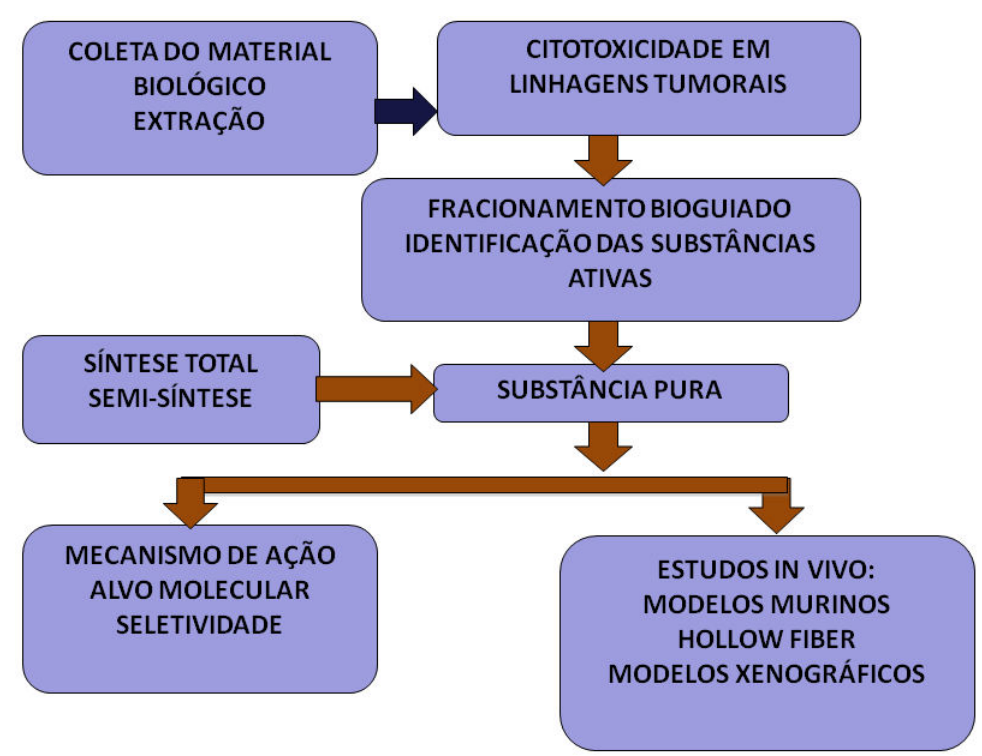

Figura 4. Fluxograma de desenvolvimento do programa de prospecção de moléculas com potencial anticâncer no Laboratório Nacional de Oncologia Experimental (LOE-UFC) 


\section{Moléculas Promissoras}

No âmbito dessas colaborações, o programa de screening do LOE, no período de 2000 a 2007, já avaliou a citotoxicidade de 5166 amostras, das quais 358 foram consideradas interessantes quanto ao potencial anticâncer. A seguir são apresentados os resultados já obtidos com três moléculas selecionadas no programa de prospecção de moléculas anticâncer do LOE-UFC, a piplartina (17), a biflorina (18) e o pisosterol (19).

A piplartina (Figura 5), também conhecida como piperlongumina, é um alcalóide/amida encontrado em espécies do gênero Piper, tais como $P$. longum $L$. (pimenta longa), $P$. tuberculatum L. (pimenta d'água), P. asborescens Roxb. (Pimenta do fruto ganchoso), $P$. callosum Ruiz \& Pav., $P$. retrofctum Vahl. e $P$. sylvaticum Roxb. ${ }^{33}$ Nos estudos conduzidos pelo nosso grupo em colaboração com o grupo do Prof. Edilberto Rocha Silveira do Departamento de Química Orgânica e Inorgânica da UFC, a piplartina foi obtida das Raízes de $P$. tuberculatum (Figura 5) num procedimento simples que envolve apenas 2 etapas, com rendimento de $10,4 \%$.

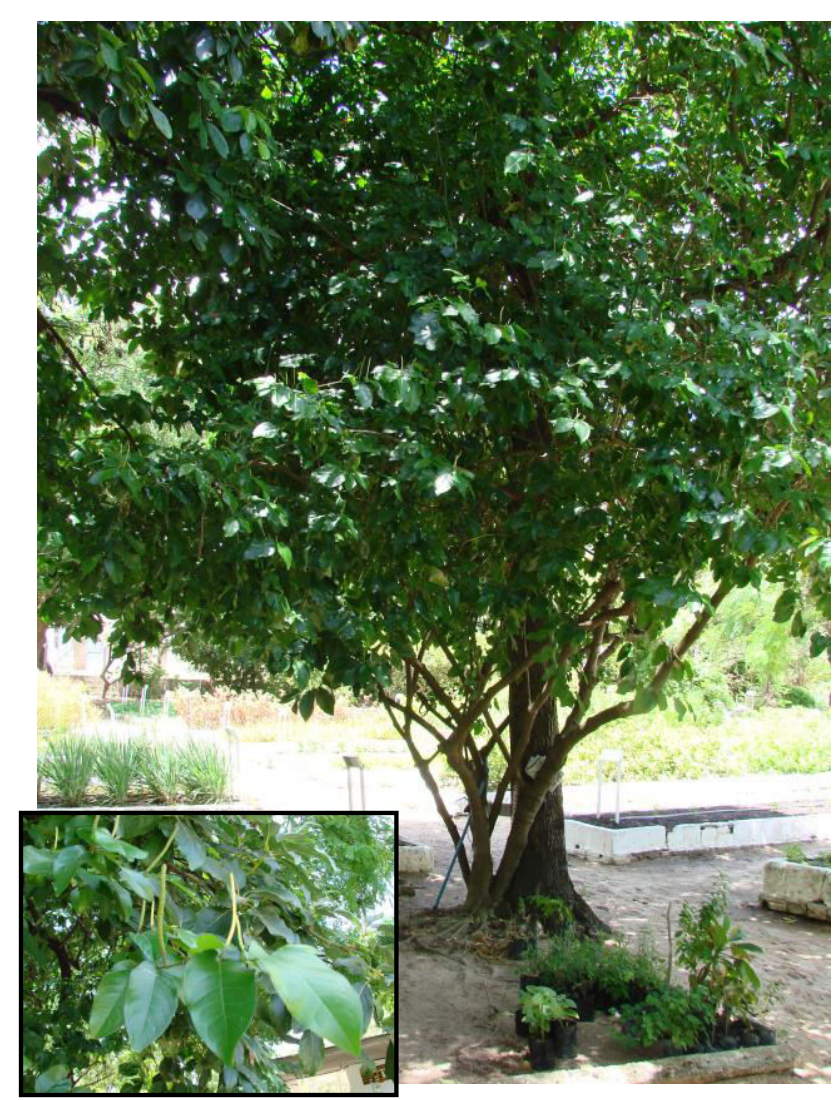

Figura 6. Fotografia de um exemplar de Piper tuberculatum L., mostrando no detalhe os frutos da planta. Foto: Prof. Dr. Edilberto Rocha da Silveira
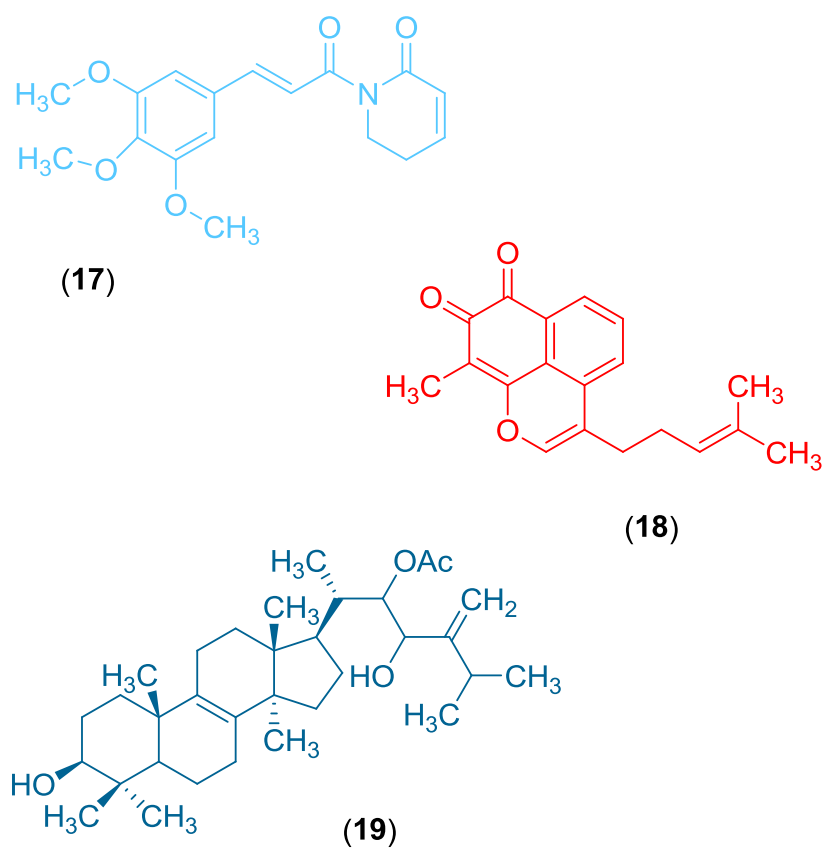

(18)

Figura 5. Moléculas selecionadas no programa de prospecção de moléculas com potencial anticâncer do no Laboratório Nacional de Oncologia Experimental (LOE-UFC)

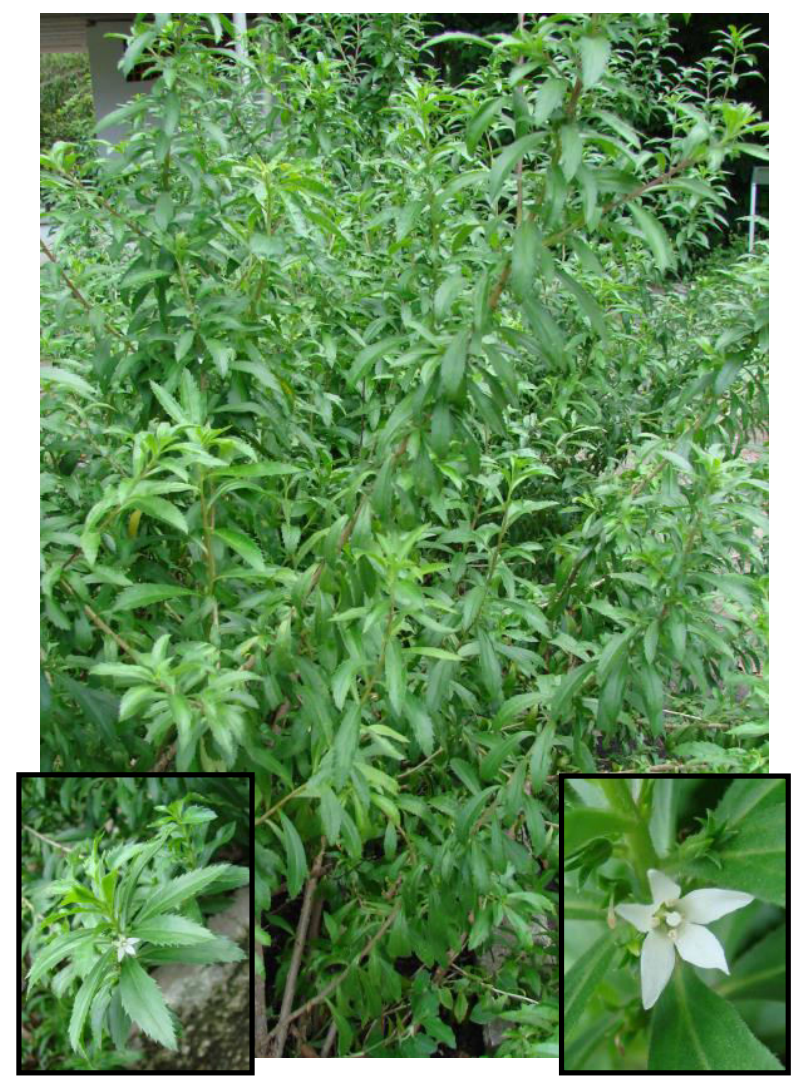

Figura 7. Fotografia de um exemplar de Capraria biflora L., mostrando no detalhe a flor da planta. Foto: Prof. Dr. Edilberto Rocha da Silveira 
Esta substância apresenta diversas atividades biológicas, incluindo atividade citotóxica e antitumoral. A piplartina apresentou potente atividade citotóxica em todas as linhagens tumorais testadas. ${ }^{34-37}$ Por comparação da citotoxicidade de moléculas com estruturas relacionadas com a piplartina, foi identificado que a presença da carbonila $\alpha, \beta$-insaturada do anel amídico é essencial para a sua atividade citotóxica. Em células mononucleares de sangue periférico de doadores saudáveis expostas a piplartina, foi observada apenas fraca atividade citotóxica. Com relação ao seu mecanismo de ação, a piplartina induziu apoptose em células leucêmicas HL-60, com participação da via intrínseca, de maneira dependente da concentração, como observado pelo padrão de morfologia celular, integridade da membrana citoplasmática, alteração no potencial transmembrânico da mitocôndria e aumento da fragmentação do DNA. Na análise do ciclo celular, foi observado bloqueio na fase $\mathrm{G}_{2}{ }^{38,39} \mathrm{~A}$ piplartina foi capaz de induzir dano ao DNA em células V79, como observado pelo ensaio do cometa alcalino e neutro. ${ }^{39}$

Dando continuidade aos estudos de genotoxicidade, foram realizados experimentos em modelos procarióticos e eucarióticos. Não foi observada atividade mutagênica, com ou sem ativação metabólica (S9), nas linhagens de Salmonelas (modelo procariótico) testadas. ${ }^{40}$ Por outro lado, a piplartina foi mutagênica e recombinogênica em linhagens de Saccharomyces cerevisiae (modelo eucariótico). ${ }^{39}$ Isto pode ser explicado pela diferença fisiológica entre a enzima topoisomerase II de eucariotos e procariotos, refletindo uma possível interferência da piplartina sobre a atividade desta enzima. No ensaio do micronúcleo in vivo, a piplartina induziu aumento da freqüência de micronúcleo na maior dose testada $(100 \mathrm{mg} / \mathrm{kg})$. Entretanto, não alterou a proporção de eritrócitos policromáticos/ normocromáticos. ${ }^{40}$

No ensaio de atividade antitumoral in vivo, a piplartina mostrou eficácia em animais inoculados com Sarcoma 180, e efeitos tóxicos moderados e potencialmente reversíveis. ${ }^{41}$ Além disso, a combinação da piplartina com o 5-fluourouracil levou a um aumento da inibição do crescimento tumoral in vitro e in vivo. ${ }^{42}$ As análises hematológicas mostraram leucopenia após tratamento dos animais com o 5fluourouracil, a qual foi revertido pela combinação com a piplartina.

A Capraria biflora L. (Figura 7) é uma planta da família Scrophulariaceae amplamente distribuída nas Américas do Sul e do Norte, onde suas raízes têm propriedade antibacteriana e suas folhas são Rev. Virtual Quim. |Vol 2| |No. 1| |47-58| utilizadas popularmente para tratamento de dor, febre, resfriado, vômito, diarréia, hemorroidas, reumatismo e edema. ${ }^{43,44} \mathrm{~A}$ biflorina (Figura 5) é uma $o$-naftoquinona (6,9-dimetil-3-(4-metil-3pentenil)nafta[1,8-bc]-piran-7,8-diona) prenilada de origem natural que pode ser facilmente obtida das raízes da $C$. biflora, sendo isolada pela primeira vez em 1953 por Gonçalves de Lima e colaboradores. Seus dados de $\mathrm{RMN}$ de ${ }^{13} \mathrm{C}$ foram registrados pela primeira vez por Fonseca e colaboradores. ${ }^{45,46}$

Uma vez que as $o$-naftoquinonas constituem um grupo promissor de drogas anticâncer, nossos pesquisadores realizaram estudos da atividade citotóxica da biflorina. Os resultados mostraram que a biflorina possui potente atividade citotóxica em células tumorais in vitro e in vivo e que não estão associadas à indução de dano oxidativo, uma vez que mostrou atividade antioxidante. ${ }^{47,48}$ Estudos preliminares sobre o mecanismo de ação citotóxico da biflorina realizadas pela nossa equipe, sugere que esta molécula possui inúmeras atividades e que esta depende da origem celular. Em células leucêmicas HL60, a biflorina foi capaz de induzir diferenciação celular, enquanto que em células de melanoma B16F10 foi capaz de inibir a colonização tumoral no pulmão, mostrando um potencial anti-metastático, além de um aumento significativo na sobrevida dos animais. Além dessas atividades, a biflorina apresenta-se como uma substância eficaz e segura, visto que não apresenta potencial mutagênico. Estes estudos estão sendo conduzidos pelo Grupo da Profa. Telma Lemos do Departamento de Química Orgânica e Inorgânica da UFC.

O fungo Pisolithus tinctorius pertence à classe dos Basidiomycetes, da família das Sclerodermateceae, é um fungo muito bem aproveitado na agricultura sendo encontrado nas raízes e solo que circundam muitas espécies de pinheiros e eucaliptos, formando ectomicorrizas. ${ }^{49}$ Estas tem a função de proteger pinheiros e/ou eucaliptos contra doenças causadas por microorganismos, e no processo de reflorestamento, estimulando o crescimento da planta, mesmo em solos de baixa fertilidade devido à produção de fitohormônios. ${ }^{50}$

Ensaios microbiológicos avaliando a atividade do extrato bruto do $P$. tinctorius demonstraram atividade microbicida. ${ }^{51}$ Dessa forma, inúmeros compostos já foram isoladas do $P$. tinctorius sendo os triterpenos, os constituintes majoritários. ${ }^{52-54}$ Dentre esses, o pisosterol $\quad(3 \beta, 22,23$-tri-idroxi-22-acetoxi-24-metillanosta-8,24(28)-dieno) (Figura 5) vem sendo estudado pelo nosso grupo em colaboração com o Prof. Manoel Andrade-Neto do Departamento de Química Orgânica e Inorgânica da UFC desde 2003. O 
procedimento de extração e purificação do pisosterol é simples, envolve apenas uma etapa de purificação em cromatografia em sílica gel do material extraído com acetona, e possui um bom rendimento $(5,7 \%)$.

Os primeiros estudos demonstraram que 0 pisosterol possui atividade citotóxica em células tumorais in vitro, sem, no entanto, alterar o desenvolvimento embrionário do ouriço do mar ou causar lise de eritrócitos de camundongos. ${ }^{55}$ Estudos com Sarcoma 180 demonstraram que o pisosterol também inibe o crescimento tumoral in vivo. ${ }^{56}$ Concomitantemente, foi observado que o pisosterol é um potente indutor de diferenciação celular, levando as células leucêmicas HL-60 a diferenciação monocítica, sendo este um dos seus prováveis mecanismos de ação. ${ }^{57}$

Várias moléculas sinalizadoras e suas vias de ativação/inibição estão correlacionadas com a indução de diferenciação celular, dentre elas a proteína quinase $C$ (PKC), MAPK (mitogen-activated protein kinase) tais como ERK $1 / 2,{ }^{58} \mathrm{c}^{-M Y C}{ }^{59}$ entre outros. Inúmeras linhagens tumorais apresentam amplificação do $c-m y c{ }^{60}$ principalmente na linhagem leucêmica $\mathrm{HL}-60 .^{61,62} \mathrm{O}$ oncogene $c$-myc está relacionado a uma série de eventos celulares, dentre eles proliferação, diferenciação, invasão, entre outros. ${ }^{63}$ Desta forma, em 2007, demonstramos que o pisosterol diminui de $99 \%$ para $10 \%$ a amplificação gênica no cromossomo 8 (8q24) em regiões homogeneamente coradas ou HSRs (homogeneously staining regions) além de inibir a proliferação celular. ${ }^{64}$

Todos esses dados sugerem que essas substâncias apresentam um potencial anticâncer promissor, sendo necessária à conclusão do seu estudo préclínico para avaliação da sua viabilidade e segurança para realização dos ensaios clínicos de fase I. Nesse momento, a colaboração com a Indústria farmacêutica é fundamental para viabilização desses estudos e para o sucesso no desenvolvimento de um novo fármaco antineoplásico com origem na biodiversidade brasileira.

\section{Conclusões}

No Brasil, as pesquisas relacionadas às ciências básicas que lidam com a identificação e caracterização de biomoléculas com potencial terapêutico, em especial a química e a farmacologia, são pródigas em suprirem os periódicos científicos especializados com trabalhos de elevado nível científico. Entretanto, nenhuma dessas moléculas, embora promissoras, passou ainda para a etapa clínica. Enquanto isso, o país despende milhões de dólares de suas divisas na importação de fármacos antineoplásicos para suprir a indústria farmacêutica nacional. Além disso, os quimioterápicos anticâncer consomem uma significante parcela dos recursos destinados ao Sistema Único de Saúde (SUS). Portanto, é crucial e estratégico para o país que novas moléculas com comprovado potencial uso terapêutico no câncer vençam a inércia acadêmica e alcancem o mercado consumidor no mais breve período possível. O Laboratório Nacional de Oncologia Experimental (LOE) reunindo as demais competências acadêmicas do Centro de Pesquisa e Desenvolvimento de Medicamentos da UFC (CPDM) em estreita colaboração com INCT de Fármacos e Medicamentos (INCT-INOFAR) pretende viabilizar novas opções terapêuticas para o tratamento do câncer, atuando nas diversas etapas da cadeia de desenvolvimento de medicamentos, priorizando algumas moléculas (piplartina, biflorina e pisosterol) selecionadas no seu programa de bioprospeç̧ão.

\section{Referências Bibliográficas}

${ }^{1}$ Srivastava, V.; Negi, J. K.; Gupta, M.; Khanuja, S. P. S. Bioorg. Med. Chem. 2005, 13, 5892. [CrossRef]

${ }^{2}$ Sítio do Instituto Nacional do Câncer (INCA). Disponível em: <http://www.inca.gov.br>. Acesso em: 15 junho 2009.

${ }^{3}$ Kummar, V.; Abbas, A.; Fausto, N.; ROBBINS \& COTRAN - Pathology Basis of Disease, 7a. ed., WB Saunders: China, 2004.

${ }^{4}$ Newman D. J.; Cragg, G. M.; J. Nat. Prod. 2007, 70, 461. [CrossRef]

${ }^{5}$ Butler, M. S.; Nat. Prod. Rep. 2008, 25, 475. [CrossRef]

${ }^{6}$ Harvey, A. L.; Drug Discov. Today 2008, 13, 894. [CrossRef]

${ }^{7}$ Pinto, A. C.; Silva, D. H. S.; Bolzani, V. S.; Lopes, N. P.; Epifanio, R. de A.; Quim. Nova 2002, 25, 45. [CrossRef]

${ }^{8}$ De Almeida, V. L.; Leitão, A.; Reina, L. C. B.; Montanari, C. A.; Donnici, C. L.; Lopes, M. T. P. Quim. Nova 2005, 28, 118. [CrossRef]

${ }^{9}$ Hanahan, D.; Weinberg, R. A. Cell 2000, 100, 57. [CrossRef]

${ }^{10}$ Foster, I. Radiography 2008, 14, 144. [CrossRef]

${ }^{11}$ Louro, I. D.; Llerena Jr, J. C.; Vieira de Melo, M. S.; Ashton-Prolla, P.; Conforti-Fróes, N.; Genética Rev. Virtual Quim. |Vol 2| |No. 1| |47-58| 
Molecular do Câncer, 1a. ed., MSG Produção Editorial: São Paulo, 2002.

${ }^{12}$ Vermeulen, K.; Van Bockstaele, D. R.; Berneman, Z. N. Cell Prolif. 2003, 36, 131. [CrossRef]

${ }^{13}$ Newman, D. J.; Cragg, G. M. Em Anticancer Agents from Natural Products; Cragg, G.M.; Kingston, D. G. J.; Newman, D. J., eds.; CRC Press, Taylor \& Francis Group, LLC: Boca Raton, 2005, cap. 1.

${ }^{14}$ Cragg, G. M.; Newman, D. J. J. Ethnopharmacol. 2005, 100, 72. [CrossRef]

${ }^{15}$ Sítio do National Cancer Institute. Disponível em: <http://www.cancer.gov>. Acesso em: 15 junho 2009

${ }^{16}$ Bailly, C. Biochem. Pharmacol. 2009, 77, 1447. [CrossRef]

${ }^{17}$ Schwartsmann, G.; Workman, P. Eur. J. Cancer 1993, 29: 3. [CrossRef]

${ }^{18}$ Newman, D. J.; Cragg, G. M.; Snader, K. M.; J. Nat. Prod. 2003, 66, 1022. [CrossRef]

${ }^{19}$ Cragg, G. M.; Newman, D. J.; Yang, S. S. J. Nat. Prod. 2006, 69, 488. [CrossRef]

${ }^{20}$ Singh, S. B.; Barrett, J. F. Biochem. Pharmacol. 2006, 71, 1006. [CrossRef]

${ }^{21}$ Zhang, J. T. Therapie 2002, 57, 2137. [PubMed]

${ }^{22}$ Cragg, G. M.; Grothaus, P. G.; Newman, D. J. Chem. Rev. 2009, 109, 3012. [CrossRef]

${ }^{23}$ Elisabetsky, E.; Costa-Campos, L. J. Ethnopharmacol. 1996, 51, 111. [CrossRef]

${ }^{24}$ Kato, M. J. Phytochemistry 2001, 57, 621. [CrossRef]

${ }^{25}$ Costa-Lotufo, L. V.; Montenegro, R. C.; Moraes, M. O.; Pessoa, O. D. L.; Silveira, E. R. Pessoa, C. Em Botanical Medicine in Clinical Pratice; Watson, R. R.; Preedy, V. R., eds.; CAB International: Cambridge, 2008, cap. 29.

${ }^{26}$ Costa-Lotufo, L. V.; Pessoa, C.; Moraes, M. O.; Pessoa, O. D. L.; Silveira, E.R. Em Recent Progress in Medicinal Plants, vol. 11, Drug Development from molecules. Govil, J.N.; Singh, V.K.; Arunachalam, C., eds.; Studium Press, LLC: Houston, 2006, cap. 3.

${ }^{27}$ Pessoa, C.; Costa-Lotufo, L. V.; Leyva, A.; Moraes, M. E. A.; Moraes, M. O. Em Lead Molecules from Natural Products: Discovery and New Trends. Khan, M.; Ather, A., eds.; Elsevier: Amsterdan, 2006, cap. 12.

${ }^{28}$ Bolzani, V. S.; Young, M. C. M.; Furlan, M.; Cavalheiro, A. J.; Araújo, A. R.; Silva, D. H. S.; Lopes, M. N. An. Acad. Bras. Ci. 1999, 71, 181.

${ }^{29}$ Mans, D. R. A.; Rocha, A. B.; Schwartsmann, G. Oncologist 2000, 5: 185. [CrossRef]

Rev. Virtual Quim. |Vol 2| |No. 1| |47-58|
${ }^{30}$ Monks, N. R.; Bordingnon, S. A. L.; Ferraz, A.; Machado, K. R.; Faria, D. H.; Lopes, R. M.; Mondin, C. A.; Souza, I. C. C.; Lima, M. F. S.; Rocha, A. B.; Schwartsmann, G. Pharm. Biol. 2002, 40, 603. [CrossRef]

${ }^{31}$ Monks, N. R., Ferraz, A.; Bordignon, S.; Machado, K. R.; Lima, M. F. S.; Rocha, A. B.; Schwartsmann, G. Pharm. Biol. 2002, 40, 494. [CrossRef]

32 Mosmann, T. J. Immunol. Methods 1983, 16, 55. [CrossRef]

${ }^{33}$ Bezerra, D. P.; Pessoa, C.; Pessoa, O. D. L.; Lima, M. A. S.; Moraes, M. O.; Costa-Lotufo, L. V. Em, Recent Progress in Medicinal Plants: Standardization of Herbal/Ayurvedic Formulations; Govil, J. N.; Singh, V. K.; Arunachalam, C., eds.; Studium Press, LLC: Houston, 2008.

${ }^{34}$ Duh, C. Y.; Wu, Y. C.; Wang, S. K. J. Nat. Prod. 1990, 53, 1575. [CrossRef]

${ }^{35}$ Duh, C. Y.; Wu, Y. C.; Wang, S. K. Phytochemistry 1990, 29, 2689. [CrossRef]

${ }^{36}$ Bezerra, D. P.; Pessoa, C.; Moraes, M. O.; Silveira, E. R.; Lima, M. A. S.; Elmiro, F. J. M.; Costa-Lotufo, L. V. Z. Naturforsch. 2005, 60C, 539. [PubMed]

${ }^{37}$ Kong, E. H.; Kim, Y. J.; Kim, Y. J.; Cho, H. J.; Yu, S. N.; Kim, K. Y.; Chang, J. H.; Ahn, S. C. Oncol. Rep. 2008, 20, 785. [CrossRef]

${ }^{38}$ Bezerra, D. P.; Militão, G. C. G.; Castro, F. O.; Pessoa, C.; Moraes, M. O.; Silveira, E. R.; Lima, M. A. S.; Elmiro, F. J. M.; Costa-Lotufo, L. V. Toxicol. in Vitro 2007, 21, 1. [CrossRef]

${ }^{39}$ Bezerra, D. P.; Pessoa, C.; Moraes, M. O.; Alencar, N. M.; Mesquita, R. O.; Lima, M. W.; Alves, A. P.; Pessoa, O. D.; Chaves, J. H.; Silveira, E. R.; CostaLotufo, L. V. J. Appl. Toxicol. 2008, 28, 599. [CrossRef]

${ }^{40}$ Bezerra, D. P.; Vasconcellos, M. C.; Machado, M. S.; Villela, I. V.; Rosa, R. M.; Moura, D. J.; Pessoa, C.; Moraes, M. O.; Silveira, E. R.; Lima, M. A. S.; Aquino, N. C.; Henriques, J. A. P.; Saffi, J.; Costa-Lotufo, L. V. Mutat. Res. 2009, 677, 8. [CrossRef]

${ }^{41}$ Bezerra, D. P.; Castro, F. O.; Alves, A. P. N. N.; Pessoa, C.; Moraes, M. O.; Silveira, E. R.; Lima, M. A. S.; Elmiro, F. J. M.; Costa-Lotufo, L. V. Braz. J. Med. Biol. Res. 2006, 39, 801. [CrossRef]

42 Bezerra, D. P.; Moura, D. J.; Rosa, R. M.; Vasconcellos, M. C.; Silva, A. C. R.; Silveira, E. R.; Moraes, M. O.; Henriques, J. A. P.; Costa-Lotufo, L. V.; Saffi, J. Mutat. Res. 2008, 652, 164.[CrossRef]

${ }^{43}$ Serpa, J. Anais da Faculdade de Medicina Universidade de Recife 1958, 18, 275. 
${ }^{44}$ Corrêa, M. P.; Dicionário das Plantas Úteis do Brasil e das Exóticas Cultivadas vol. 2, 1a. ed., Imprensa Nacional: Rio de Janeiro, 1984.

${ }^{45}$ Gonçalves de Lima, O.; D’Albuquerque, I. L.; Loureiro, P.; Carmona, C. I.; Bernard, M. Z. Rev. Quim. Ind. 1953, 22, 1.

${ }^{46}$ Fonseca, A. M.; Silveira, E. R.; Pessoa, O. D. L.; Lemos, T. L. G. Magn. Res. Chem. 2002, 41, 1038. [CrossRef]

${ }^{47}$ Vasconcellos, M. C.; Montenegro, R. C.; Militão, G. C. G.; Pessoa, O. D.; Fonseca, A. M.; Lemos, T. G. L.; Pessoa, C.; Moraes, M. O.; Costa-Lotufo, L. V. Z. Naturforsch. 2005, 60C 394. [PubMed]

${ }^{48}$ Vasconcellos, M. C.; Bezerra, D. P.; Fonseca, A. M.; Pereira, M. R. P.; Lemos, T. G. L.; Pessoa, O. D.; Pessoa, C.; Moraes, M. O.; Alves, A. P. N. N.; CostaLotufo, L. V. Biol. Pharm. Bull. 2007, 30, 1416. [CrossRef]

${ }^{49}$ Baumert, A.; Schumann, B.; Porzel, A.; Schmidt, J.; Strack, D. Phytochemistry 1997, 45, 499. [CrossRef]

${ }^{50}$ Arshad, M.; Frankenberger Jr., W. T. Plant and Soil 1991, 133, 1. [CrossRef]

${ }^{51}$ Tsantrizos, Y. S.; Kope, H. H.; Fortin, J. A.; Ogilvie, K. K. Phytochemistry 1991, 30, 1113. [CrossRef]

${ }^{52}$ Gill, M.; Kiefel, M. J.; Skelton, B. W.; White, H. Aust. J. Chem. 1989, 42, 995. [CrossRef]

${ }^{53}$ Fujimoto, H.; Nakayama, M.; Nakayama, Y.; Yamazaki, M.; Chem. Pharm. Bull. 1994, 42, 694. [PubMed]

${ }^{54}$ Baumert, A.; Schumann, B.; Porzel, A.; Schmidt, J.; Strack, D. Phytochemistry 1997, 45, 499. [CrossRef]

${ }^{55}$ Montenegro, R. C.; Jimenez, P. C.; Feio Farias, R. A.; Andrade-Neto, M.; Silva Bezerra, F.; Moraes, M. E. A.; Pessoa, C.; Moraes, M. O.; Costa-Lotufo, L. V. Z. Naturforsch. 2004, 59C, 519. [PubMed]

${ }^{56}$ Montenegro, R. C.; Farias, R. A. F., Pereira, M. R. P.; Alves, A. P. N. N.; Bezerra, F. S.; Andrade-Neto, M; Pessoa, C.; Moraes, M. O.; Costa-Lotufo, L. V. Biol. Pharm. Bull. 2008, 31, 454. [CrossRef]

${ }^{57}$ Montenegro, R. C.; Vasconcellos, M. C.; Silva Bezerra, F.; Andrade-Neto, M.; Pessoa, C.; Moraes, M. O.; Costa-Lotufo, L. V. Toxicol. in Vitro 2007, 21, 795. [CrossRef]

${ }^{58}$ Dent, P.; Yacoub, A.; Fisher, P. B.; Hagan, M. P.; Grant, S. Oncogene 2003, 22, 5885. [CrossRef]

${ }^{59}$ Gandarillas, A. \& Watt, F. M. Genes Dev. 1997, 11, 2869. [CrossRef]

${ }^{60}$ Eva, A.; Robbins, K. C.; Andersen, P. R.; Srinivasan,
A.; Tronick, S. R.; Reddy, E. P.; Ellmore, N. W.; Galen, A. T.; Lautenberger, J. A.; Papas, T. S.; Westin, E. H.; Wong-Staal, F.; Gallo, R. C.; Aaronson, S. A. Nature 1982, 295, 116. [CrossRef]

${ }^{61}$ Westin, E. H.; Wong-Staal, F.; Gelmann, E. P.; Dalla Favera, R.; Papas, T. S.; Lautenberger, J. A.; Eva, A.; Reddy, E. P.; Tronick, S. R. Proc. Nat. Acad. Sci. U.S.A. 1982, 79, 2490. [CrossRef]

${ }^{62}$ Favera, R. D.; Wong-Staal, F.; Gallo, R. C. Nature 1982, 299, 61. [CrossRef]

${ }^{63}$ Henriksson, M.; Luscher, B. Adv. Cancer Res. 1996, 68, 109. [CrossRef]

${ }^{64}$ Burbano, R. R.; Lima, P. D.; Bahia, M. O.; Khayat, A. S.; Silva, T. C.; Bezerra, F. S.; Andrade Neto, M.; Moraes, M. O.; Montenegro, R. C.; Costa-Lotufo, L. V.; Pessoa, C. Cell Biol Toxicol. 2009, 25, 245. [CrossRef] 